\title{
Alocação de Carteiras Sujeitas a Risco de Crédito
}

\author{
Rogerio de Deus Oliveira ${ }^{1}$
}

\section{Caio Ibsen Rodrigues de Almeida ${ }^{2}$}

\section{Resumo}

Risco de crédito representa uma dimensão importante de risco a ser considerada por bancos quando realizam empréstimos. Em particular, em mercados emergentes as taxas de inadimplência em produtos de empréstimos bancários são muito altas, demandando maior cuidado com relação ao gerenciamento deste risco. Usualmente o risco de crédito de carteiras que envolvem empréstimos é obtido a partir de simulações de Monte Carlo de alto custo computacional que consideram diferentes fatores estocásticos relacionados a incerteza dos pagamentos dos passivos associados aos devedores. Neste trabalho, sob determinadas hipóteses, nós derivamos fórmulas fechadas para as distribuições de probabilidades das taxas de inadimplência de produtos bancários envolvendo um número grande de clientes. O conhecimento de tais distribuições nos permite calcular com facilidade o risco de crédito associado a cada produto oferecido pelo banco. Adicionalmente, a partir destas distribuições, utilizando Retorno Total como medida de retorno, e Perda Máxima como medida de risco, resolvemos o problema de obtenção de carteiras ótimas envolvendo risco de crédito.

Palavras chave: Inadimplência, risco de crédito, distribuição de probabilidades, alocação de carteiras, retorno total, perda máxima, valor em risco .

JEL Code: C65, G11.

\footnotetext{
${ }^{1}$ JPMorgan Chase \& CO, NY.

${ }^{2}$ IBMEC-RJ e Departamento de Matemática, Stanford University
}

\begin{tabular}{lllllllllll}
\hline Revista & Brasileira & de & Finanças & v. & 1, & $\mathrm{n}^{\underline{\underline{O}}}$ & 2, & pp.301-339 & Dezembro & 2003 \\
\hline
\end{tabular}




\begin{abstract}
Credit Risk is an important dimension to be considered in the risk management procedures of financial institutions. It is particularly useful in emerging markets where default rates on bank loan products are usually high. It is usually calculated through highly costly Monte Carlo simulations which consider different stochastic factors driving the uncertainty associated to the borrowers' liabilities. In this paper, under some restrictions, we derive closed form formulas for the probability distributions of default rates of bank loans products involving a big number of clients. This allows us to quickly obtain the credit risk of such products. Moreover, using these probability distributions, we solve the problem of optimal portfolio allocation under default risk.
\end{abstract}

\title{
1. Introdução.
}

Risco de crédito é provavelmente o risco mais antigo que os bancos conhecem e procuram gerenciar. Todavia, apenas recentemente ele foi entendido de forma mais ampla, levando em consideração o conceito de carteiras de ativos. CreditMetrics do JP Morgan e CreditRisk+ do CSFB estão entre os modelos mais populares desenvolvidos nos últimos anos para analisar o risco de crédito de forma agregada.

Estes modelos têm limitações quando aplicados a alguns produtos bancários, especialmente em mercados emergentes. Por exemplo, CreditRisk + assume a hipótese básica de que o número de inadimplências pode ser aproximado por uma distribuição de Poisson. Esta aproximação é razoável quando a probabilidade de inadimplência de cada indivíduo é pequena, digamos menor que $5 \%$. No entanto, os sistemas financeiros de mercados emergentes têm atividades tais como crédito ao consumidor, leasing e cartão de crédito com taxas de inadimplência que freqüentemente excedem 10\%. Nestas situações não é razoável assumir esta hipótese chave. Por outro lado, CreditMetrics utiliza simulação de Monte Carlo sempre que existe correlação entre os riscos de inadimplência de dois ou mais devedores. O risco de crédito dos produtos bancários mencionados acima tem alta correlação devido a fatores macroeconômicos tais como desemprego, demanda agregada, etc. Isto diretamente implica que a dimensão da simulação de Monte Carlo acaba sendo igual ao número de clientes, muito alta para ser factível.

A metodologia desenvolvida neste trabalho supera estas limitações. Derivamos fórmulas analíticas para a distribuição de probabilidade das taxas de inadimplência de um único produto bancário quando existe um número muito grande de pequenos clientes. Conseqüentemente, a simulação de Monte Carlo de alta dimensão é evitada e o risco de crédito agregado pode ser explicitamente computado. 
Quando agregamos o risco de crédito de vários produtos bancários diferentes, é melhor trabalhar com um conceito de risco alternativo ao já consagrado Valor a Risco (VAR), chamado de Perda Máxima (PM). A metodologia PM foi recentemente desenvolvida e tem interesses e vantagens próprias. Basicamente, esta metodologia determina a perda máxima que um portfólio pode sofrer quando o fator de risco é limitado a um conjunto com probabilidade igual a 99\%. Quando os retornos são lineares no fator de risco, as metodologias PM e VAR coincidem. O leitor interessado é remetido a Studer (1995) para uma descrição detalhada e o desenvolvimento completo da metodologia PM.

Para o nosso problema em questão, PM é melhor do que VAR porque, como será demonstrado, a distribuição de probabilidade dos retornos do portfólio agregado apresenta assimetria, cauda grossa e é multi modal. Portanto, a volatilidade não será necessariamente uma boa medida de risco e, para o cômputo do VAR, seria necessário recorrer à simulação de Monte Carlo, com todo o custo computacional associado. Por outro lado, PM é um programa de otimização que possui algoritmos para solução numérica eficiente e acurada.

Além disso, o VAR calculado por simulação de Monte Carlo se presta apenas a soluções numéricas. Por outro lado, o conceito PM de risco nos fornece condições de primeira ordem e de envelope que permitem demonstrar muitas propriedades e resultados qualitativos para a fronteira de portfólios ótimos. Como resultado, veremos que os portfólios ótimos do problema em questão compartilham várias (mas não todas) propriedades importantes dos portfólios ótimos da otimização clássica média-variância de Markowitz.

Por fim, desenvolvemos uma versão de CAPM para portfólios de crédito. Com isso, obtemos implicações empiricamente testáveis para o nosso modelo. Todavia, postergamos um efetivo teste econométrico para futuras extensões do trabalho. Em primeiro lugar porque é notória a dificuldade de obter bancos de dados de qualidade com informações agregadas para ativos de crédito. Em segundo lugar, a implicação econométrica que obtemos é bastante simplória e passível dos mesmos problemas conceituais e dificuldades econométricas associados aos testes tradicionais de CAPM. Existe toda uma extensa literatura destinada a contornar estes problemas e aperfeiçoar os testes empíricos de CAPM - ver por exemplo Blume e Friend (1973), Fama e MacBeth (1972 , 1973) e Black, Jensen e Scholes (1972), entre outros. Neste artigo, pretendemos apenas iniciar o potencialmente longo e detalhado tema dos testes empíricos.

A Seção 2 apresenta o modelo envolvendo um único produto de 
empréstimo bancário, onde a distribuição de probabilidades da taxa de inadimplência neste produto é obtida sob a hipótese de que a inadimplência de cada cliente apresenta pequena influência sobre a taxa de inadimplência final do produto. Já a Seção 3, generaliza o problema considerando diferentes produtos bancários e formulando o problema de otimização de carteiras do ponto de vista do banqueiro, que gostaria de identificar a fronteira eficiente dos produtos que estão sendo oferecidos por seu banco. A seção 4 apresenta a solução do problema de otimização proposto na seção anterior. A seção 5 resolve novamente o problema de obtenção da carteira ótima considerando na otimização o ativo livre de risco. A seção 6 apresenta considerações econométricas descritas no parágrafo anterior. E por último, a seção 7 apresenta comentários e conclusões. Os apêndices apresentam as contas e demonstrações de resultados discutidos ao longo do texto.

\section{O modelo para um único produto bancário.}

Neste trabalho será analisado somente o risco de crédito de produtos bancários onde há um número muito grande de pequenas exposições individuais. A inadimplência de um único cliente é irrelevante para o retorno do portfólio. Conseqüentemente, a única varivel aleatória importante é a taxa de inadimplência de cada produto. Inicialmente analisaremos a taxa de inadimplência de uma única atividade bancária, digamos crédito ao consumidor. Posteriormente passaremos à análise do risco de crédito combinado de múltiplas atividades.

Seja $X_{i}$ a variável aleatória que reflete a solidez econômica do cliente $i$. Suponha

i) $X_{i}$ depende linearmente de um choque macroeconômico $Z$ (por exemplo desemprego) e um específico $\varepsilon_{i}$, com pesos independentes do cliente. Isto é, $X_{i}=a . Z+b \varepsilon_{i}$,

ii) $\left\{Z,\left(\varepsilon_{i}\right)_{i=1,2, \ldots}\right\}$ são independentes e têm distribuição normal com média zero e variância igual a 1 ,

iii) $X_{i}$ tem distribuição normalizada. Isto é, $a^{2}+b^{2}=1$,

iv) a correlação entre o risco de crédito de dois clientes quaisquer é $\rho$. Isto , $\operatorname{Corr}\left(X_{i}, X_{j}\right)=a^{2}=\rho$.

Com estas hipóteses $X_{i}$ têm distribuição normal padrão e podemos escrever

$$
X_{i}=\sqrt{\rho Z}+\sqrt{1-\rho \varepsilon_{i}} .
$$


A inadimplência do cliente $i$ ocorre quando $X_{i} \leq \bar{x}$, para um certo nível crítico $\bar{x}$. Como o nível crítico é o mesmo para todos os clientes, estamos implicitamente assumindo que todos eles têm o mesmo perfil de risco. Não sendo este o caso, podemos agrupar os indivíduos em grupos diferentes sendo cada grupo composto de clientes com perfis de risco homogêneos. O processo de agregar o risco de crédito de cada grupo diferente é virtualmente idêntico ao processo de agregar o risco de crédito de produtos diferentes, o que será detalhado mais adiante.

O valor de $\bar{x}$ é obtido resolvendo a equação $\Phi(\bar{x})=p_{d}$, onde $\Phi($.$) é a$ função de probabilidade acumulada da distribuição normal padronizada e $p_{d}$ é a probabilidade de um cliente individual inadimplir, que deve ser estimada utilizando modelos de credit score ou taxas históricas de inadimplência.

Defina $Y_{n}$ como sendo a taxa amostral de inadimplência, isto é

$$
\begin{gathered}
Y_{n}=\frac{\chi_{\left[X_{1} \leq \bar{x}\right]}+\chi_{\left[X_{2} \leq \bar{x}\right]}+\ldots \chi_{\left[X_{n} \leq \bar{x}\right]}}{n}, \text { onde } \\
\chi_{\left[X_{i} \leq \bar{x}\right]}=\left\{\begin{array}{lll}
1 & \text { se } X_{\left[X_{i} \leq \bar{x}\right]} \\
0 & \text { se } X_{\left[X_{i}>\bar{x}\right]}
\end{array}\right.
\end{gathered}
$$

Caso a inadimplência individual de qualquer cliente tenha impacto relevante sobre o retorno do portfólio, como é normalmente o caso de portfólios de empréstimos corporativos, se faz mister a utilização de simulação de Monte Carlo $n$-dimensional. No entanto, $n$ costuma ser muito grande apenas para produtos bancários onde a inadimplência individual é irrelevante. Neste caso a única variável aleatória importante é $Y$, a taxa populacional de inadimplência, definida como o limite em distribuição, quando o número de clientes vai para infinito, de $Y_{n}$.

No caso de correlação zero, é fácil notar, pela lei dos grandes números (Shiryaev [1996] - página 325), que este limite é a constante $p_{d}$. Neste caso, não haveria nenhum risco nesta atividade bancária. No entanto, quando há correlação positiva, a lei dos grandes números não pode ser invocada, $Y_{n}$ não converge para um limite fixo, $Y$ tem uma distribuição de probabilidade não degenerada, e há risco nesta atividade bancária. No apêndice I demonstramos o seguinte resultado.

Teorema 1:

$$
Y_{n} \stackrel{D}{\rightarrow} Y \equiv \Phi\left(\frac{\bar{x}-\sqrt{\rho Z}}{\sqrt{1-\rho}}\right)
$$

onde $\stackrel{D}{\rightarrow}$ denota convergência em distribuição e $\Phi($.$) é a função de probabi-$ lidade acumulada da distribuição normal padrão. 
Observe que para $\rho=0$, o teorema 1 implica que $Y$ tem distribuição degenerada com toda a massa concentrada em $Y=\Phi(\bar{x})=p_{d}$, em linha com o argumento da lei dos grandes números utilizado acima.

No outro extremo, em caso de correlação perfeita $(\rho=1)$ a carteira se comporta como se fosse um único cliente. Neste caso apenas duas situações são possíveis: ou todos os clientes se tornam inadimplentes ou todos honram suas dívidas. Observando a equação (3) é fácil observar que uma fração $\Phi(\bar{x})$ da massa está concentrada em $Y=1$ e uma fração $1-\Phi(\bar{x})$ está concentrada em $Y=0$.

Supondo uma taxa média de inadimplência $\Phi(\bar{x})=12 \%$, as distribuições de probabilidade para valores intermediários $\rho=0.1$ e $\rho=0.9$ estão grafadas abaixo. 
Gráfico 1. Distribuição das taxas de inadimplência para diferentes valores de auto-correlação $\rho$.

O valor esperado de $Y$ é $\Phi(\bar{x})$, independentemente do valor da correlação ${ }^{3}$. Observe no gráfico que quanto maior a correlação, maior a dispersão da massa ao redor da média, o que gera mais risco. Assumindo uma taxa de recuperação de $R$, um spread $S$ e uma taxa de juros sem risco de $r$, o retorno $W$ desta classe de ativos é

$$
W=(1-Y)(1+r+S)+Y R-1=r+S-(1+r+S-R) Y
$$

${ }^{3} \mathrm{O}$ leitor é remetido ao Apêndice II onde calculamos o momento de ordem $n$ da variável aleatória $Y$. 
No Apêndice III, calculamos o VAR ao nível de $\alpha \%$ de confiança de um único produto bancário, assumindo duas distribuições limites diferentes para a taxa de inadimplência:

i) Na primeira suponha $Y$ distribuído de acordo com a equação (3), com o fator macroeconômico $Z$ tendo distribuição normal padrão. Esta distribuição para $Y$ nos fornece um VAR com nível de confiança de $1 \%$ dado por

$$
\operatorname{VAR} 1 \%=(R-S-r-1)\left(\Phi\left(\frac{\bar{x}+2,32 \sqrt{\rho}}{\sqrt{1-\rho}}\right)-\Phi(\bar{x})\right)+S+r .
$$

ii) Na segunda, aproxime $Y$ pela distribuição normal cujos primeiro e segundo momentos sejam iguais aos respectivos momentos da distribuição dada por (3). Neste caso teremos um risco de (ver apêndice III).

$$
\begin{aligned}
& \text { Normal VAR1 } \%=(R-S-r-1) . \\
& \qquad\left(\phi(\bar{x})+2.32 \sqrt{\phi_{2}(\bar{x}, \bar{x}, \rho)-\phi(\bar{x})^{2}}\right)+S+r,
\end{aligned}
$$

onde $\phi($.$) é a função de densidade da distribuição normal padrão e \phi_{2}(., ., \rho)$ a função de densidade da distribuição normal bivariada padrão com correlação $\rho$.

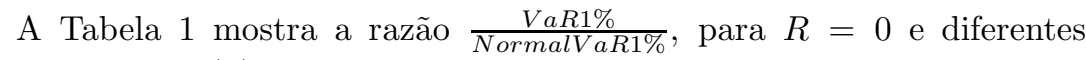
valores de $\rho$ e $\Phi(\bar{x})$. Pela tabela vemos que o erro incorrido utilizando a aproximação normal pode assumir valores consideráveis. 
Tabela 1. Razão VAR/NormalVAR

para diferentes auto-correlações

\begin{tabular}{lccc}
\multicolumn{3}{c}{ Razão } \\
\hline \hline$\rho$ & $\Phi(\bar{x})=5 \%$ & $\Phi(\bar{x})=12 \%$ & $\Phi(\bar{x})=20 \%$ \\
0.1 & 1.4681 & 1.3250 & 1.2191 \\
0.3 & 1.9654 & 1.5210 & 1.3224 \\
0.5 & 2.1663 & 1.6227 & 1.3304 \\
0.7 & 2.1663 & 1.6311 & 1.2389 \\
0.9 & 2.2883 & 1.4285 & 1.0372
\end{tabular}

Obviamente qualquer tentativa futura de aplicação econométrica deste modelo requer uma estimação de parâmetro $\rho$. Isto pode ser obtido construindo um modelo de Credit Score, efetuando uma regressão Probit e incluindo entre os regressores uma variável macroeconômica normalizada. Pela equação (1) o coeficiente associado a este regressor macroeconômico é $-\sqrt{\rho} / \sqrt{1-\rho}$. Com esta informação podemos inferir uma estimativa para $\rho$.

No mundo real não há portfólios com um número infinito de clientes com o mesmo perfil de risco e as inadimplências individuais não são completamente irrelevantes. Portanto, é razoável perguntar quantos indivíduos o portfólio deve ter para a aproximação pelo limite fazer sentido. Usando a Lei dos Grandes Desvios demonstramos no Apêndice I um resultado mais forte que o Teorema 1.

$\underline{\text { Teorema 2: }} Y_{n} \stackrel{P}{\rightarrow} Y \equiv \Phi\left(\frac{\bar{x}-\sqrt{\rho Z}}{\sqrt{1-\rho}}\right)$ com uma taxa de convergência exponencial.

onde $\stackrel{P}{\rightarrow}$ denota convergência em probabilidade (que implica convergência em distribuição).

Na prática, um portfólio com 1000 indivíduos, todos com exposição individual menor do que $0.5 \%$ da exposição total, tem probabilidade virtualmente igual a zero de assumir valores diferentes da distribuição limite.

\section{Portfólios com múltiplos produtos.}

Para analisar portfólios com múltiplos produtos, simplesmente considere choques macroeconômicos correlacionados afetando diferentes produtos. Por exemplo, com dois produtos diferentes rotulados 1 e 2, defina

$$
X_{i}^{1}=\sqrt{\rho_{1}} Z^{1}+\sqrt{1-\rho_{1}} \varepsilon_{i}
$$




$$
X_{i}^{2}=\sqrt{\rho_{2}} Z^{2}+\sqrt{1-\rho_{2}} \varepsilon_{i},
$$

onde assumimos que os fatores de risco macroeconômicos $Z^{1}$ e $Z^{2}$ têm correlação $\rho_{12}$. Assim, como na seção anterior, os parâmetros $\rho_{1}$ e $\rho_{2}$ são as correlações dentro de cada produto.

A hipótese de irrelevância de exposições individuais será observada na situação onde um mesmo indivíduo se torna inadimplente em múltiplos produtos simultaneamente.

Defina $Y^{1}, Y^{2}$ como as taxas populacionais de inadimplências de cada produto. Do Teorema 1 temos

$$
Y^{1}=\Phi\left(\frac{\bar{x}^{1}-\sqrt{\rho_{1}} Z^{1}}{\sqrt{1-\rho_{1}}}\right) \text { e } Y^{2}=\Phi\left(\frac{\bar{x}^{2}-\sqrt{\rho_{2}} Z^{2}}{\sqrt{1-\rho_{2}}}\right)
$$

onde níveis críticos $\bar{x}^{1}$ e $\bar{x}^{2}$ são obtidos invertendo as equações $\Phi\left(\bar{x}^{1}\right)=p_{d}^{1}$ e $\Phi\left(\bar{x}^{2}\right)=p_{d}^{2}$.

Suponha que sejam alocadas as quantidades de recursos $\alpha_{1}$ e $\alpha_{2}$ para os produtos 1 e 2, respectivamente. Assim, o retorno total (RT) é dado por

$$
\begin{aligned}
R T\left(\alpha_{1}, \alpha_{2}, Z^{1}, Z^{2}\right)= & \alpha_{1}\left(r+S_{1}-\left(1+r+S_{1}-R_{1}\right) \Phi\right. \\
& \left.\left(\frac{\bar{x}^{1}-\sqrt{\rho_{1}} Z^{1}}{\sqrt{1-\rho_{1}}}\right)\right) \\
+ & \alpha_{2}\left(r+S_{2}-\left(1+r+S_{2}-R_{2}\right) \Phi\right. \\
& \left.\left(\frac{\bar{x}^{2}-\sqrt{\rho_{2}} Z^{2}}{\sqrt{1-\rho_{2}}}\right)\right)
\end{aligned}
$$

Simulação de Monte Carlo pode ser usada para calcular o VAR deste portfólio. Por exemplo, suponha 2 produtos semelhantes com $R_{1}=R_{2}=0$, $S_{1}=S_{2}=15 \%, \Phi\left(\bar{x}_{1}\right)=\Phi\left(\bar{x}_{2}\right)=12 \%, \rho_{1}=\rho_{2}=0.1$. A tabela 2 mostra o VAR $1 \%$ para valores diferentes de $\rho_{12}$ e $\alpha_{1}=1-\alpha_{2}$. Os benefícios da diversificação são substanciais. O portfólio ótimo para este exemplo é obviamente $\alpha_{1}=\alpha_{2}=1 / 2$, porque os ativos têm a mesmas características.

Tabela 2. VAR1\% para 2 ativos semelhantes e para diferentes níveis de correlação entre produtos.

$\rho_{12}$ 


\begin{tabular}{lccc}
\hline \hline$\alpha_{1}$ & 0.9 & 0.5 & 0.1 \\
\hline 0 & 0.205 & 0.205 & 0.205 \\
0.25 & 0.198 & 0.177 & 0.158 \\
0.5 & 0.191 & 0.165 & 0.141
\end{tabular}

No caso em que os ativos têm características diferentes, o portfólio ótimo é obtido minimizando o risco sujeito a um retorno esperado prédeterminado. A otimização é infactível se o risco for computado por simulação de Monte Carlo. Por outro lado, conforme demonstramos na Tabela 1, VAR ou volatilidade não é uma aproximação razoável para o risco destes portfólios de crédito. Porém, no caso desta aproximação ser levada adiante, a análise de média-variância necessitaria do cálculo das covariâncias. Um pouco de álgebra mostra que

$$
\operatorname{Cov}\left(Y^{1}, Y^{2}\right)=\Phi_{2}\left(\bar{x}^{1}, \bar{x}^{2}, \rho_{12} \sqrt{\rho_{1} \rho_{2}}\right)-\Phi\left(\bar{x}^{1}\right) \cdot \Phi\left(\bar{x}^{2}\right)
$$

onde $\Phi_{2}(x, y, \rho)$ é a função de probabilidade acumulada da distribuição normal padrão bivariada com correlação $\rho$.

Ao invés de recorrer a esta aproximação grosseira, é conveniente usar um conceito de risco alternativo ao VAR chamado de Perda Máxima (PM). A metodologia PM determina a perda máxima que um portfólio pode ter quando os fatores de risco (neste caso $Z=\left[Z_{1}, Z_{2}\right]^{\prime}$ ) estão confinados a um conjunto $\Theta$ de probabilidade $99 \%$.

Esta metodologia alternativa de cálculo de risco foi detalhadamente estudada em Studer (1995). Este autor demonstra que as metodologias PM e VAR coincidem quando os fatores de risco têm distribuição normal e a função retorno total (RT) é linear em $z$.

Em seu artigo, Studer utiliza PM para calcular o risco de portfólios de opções e outros ativos não lineares. Para estes portfólios, é notória a imprecisão de calcular o risco através da metodologia VAR. Nestes casos, a metodologia PM é uma conveniente alternativa.

Dado a óbvia não linearidade (em $z$ ) da nossa função RT, é natural a opção de calcular o risco utilizando PM. Matematicamente temos o seguinte programa de otimização

$$
\begin{gathered}
P M\left(\alpha_{1}, \alpha_{2}\right)=\min _{z} R T\left(\alpha_{1}, \alpha_{2}, z^{1}, z^{2}\right) \\
\text { s.a. }\left[Z^{1}, Z^{2}\right] \in \Theta, \\
\\
\operatorname{e} \operatorname{Prob}(\Theta)=99 \%
\end{gathered}
$$


Como $Z=\left[Z_{1}, Z_{2}\right]^{\prime}$ têm distribuição normal bivariada, com matriz de variância-covariância $\sum=\left[\begin{array}{cc}1 & \rho_{12} \\ \rho_{12} & 1\end{array}\right], z^{\prime} \rho z$ tem distribuição Chiquadrada com 2 graus de liberdade. Assim, podemos escolher o conjunto $\Theta=\left\{z \in R^{2} \mid z \sum^{-1} z \leq \chi_{1 \%}\right\}$, onde $\chi_{1 \%}$ é o quantil $1 \%$ desta distribuição Chi-quadrada. O programa (P1) se transforma em

$$
\begin{aligned}
P M(\alpha)= & \min _{z} R T(\alpha, z) \\
& \text { s.a. } z^{\prime} \sum^{-1} z \leq \chi_{1 \%}
\end{aligned}
$$

A generalização de (7) e (P1') é óbvia para o caso onde temos $n$ ativos ou produtos bancários. Basta utilizar $\chi_{1 \%}$ como o quantil $1 \%$ da distribuição Chi-quadrada com $n$ graus de liberdade e $\sum$ a matriz $n \times n$ de variânciacovariância dos diferentes produtos.

Finalmente, o portfólio ótimo com retorno esperado $\mu$ é obtido resolvendo o programa

$$
\begin{aligned}
\operatorname{Risco}(\mu)= & \min _{\alpha}-P M(\alpha) \\
& \text { s.t. } \sum_{i} \alpha_{i}\left(r+S_{i}-\left(1+r+S_{i}-R_{i}\right) \Phi\left(\bar{x}^{i}\right)\right)=\mu \\
& \sum_{i} \alpha_{i}=1
\end{aligned}
$$

O resultado que segue garante que as condições de primeira ordem (C.P.O.) de (P2) são necessárias e suficientes para caracterizar a solução ótima.

lema: A função $P M($.$) é côncava.$

\section{Resolvendo o programa de otimização.}

O programa (P2) é mais simples do que parece. Apesar da própria função objetiva a ser minimizada ser a solução de outra otimização (a solução de (P1')), a solução pode ser simplificada utilizando as C.P.O. e o Teorema do Envelope.

As C.P.O. de (P2) são

$$
P M_{\alpha_{i}}=\lambda_{\mu}\left(r+S_{i}-\left(1+r+S_{i}-R_{i}\right) . \Phi(\bar{x})\right)+\lambda_{1},
$$

para cada ativo $i$. 
onde $\lambda_{\mu}$ e $\lambda_{1}$ são os multiplicadores de Lagrange da restrição de retorno esperado e da restrição que limita o somatório das variáveis em 1. Pelo Teorema do Envelope, a otimização (P1) nos fornece

$$
P M_{\alpha_{i}}=\left(r+S_{i}-\left(1+r+S_{i}-R_{i}\right) \Phi\left(\frac{\bar{x}^{i}-\sqrt{\rho_{i}} z^{i}}{\sqrt{1-\rho_{i}}}\right)\right) .
$$

Conseqüentemente obtemos

$$
\begin{aligned}
z^{i}= & \frac{\bar{x}^{1}}{\sqrt{\rho_{i}}}-\sqrt{\frac{1-\rho_{i}}{\rho_{i}}} \Phi^{-1} \\
& \left(\frac{\left(r+S_{i}-\lambda_{\mu}\left(r+S_{i}-\left(1+r+S_{i}-R_{i}\right) . \Phi(\bar{x})\right)-\lambda_{1}\right)}{1+r+S_{i}-R_{i}}\right) \\
\equiv & h_{i}\left(\lambda_{\mu}, \lambda_{1}\right) .
\end{aligned}
$$

As C.P.O. de (P1') são

$$
\alpha_{i} \cdot\left(1+r+S_{i}-R_{i}\right) \sqrt{\frac{\rho_{i}}{1-\rho_{i}}} \phi\left(\frac{\bar{x}^{i}-\sqrt{\rho_{i}} z^{i}}{\sqrt{1-\rho_{i}}}\right)=-\lambda_{P 1} e_{i}^{\prime} \sum^{-1} z
$$

onde $\lambda_{P 1} \geq 0$ é o multiplicador de Kuhn-Tucker da restrição em (P1'), $\phi($.$) é$ a função de densidade da distribuição normal padrão e $e_{i}$ é o vetor canônico $n$-dimensional. Utilizando a equação anterior podemos escrever

$$
\begin{aligned}
\alpha_{i} & =\lambda_{P 1} \frac{e_{i}^{\prime} \sum^{-1} h\left(\lambda_{\mu}, \lambda_{1}\right)}{\left(1+r+S_{i}-R_{i}\right) \sqrt{\frac{\rho_{i}}{1-\rho_{i}}} \phi\left(\frac{\bar{x}^{i}-\sqrt{\rho_{i}} h^{i}\left(\lambda_{\mu}, \lambda_{1}\right)}{\sqrt{1-\rho_{i}}}\right)} \\
& \equiv-\lambda_{P 1} g_{i}\left(\lambda_{\mu}, \lambda_{1}\right) .
\end{aligned}
$$

Substituindo (8) na equação de restrição do programa (P1') obtemos

$$
f_{1}\left(\lambda_{\mu}, \lambda_{1}\right)=h\left(\lambda_{\mu}, \lambda_{1}\right)^{\prime} \sum^{-1} h\left(\lambda_{\mu}, \lambda_{1}\right) \leq \chi_{1 \%} .
$$

Porém, a inequação (10) é, na verdade, uma equação. Caso contrário teríamos $\lambda_{P 1}=0$ e, por (9), $\alpha_{i}=0$ para todo $i$. Isto é um absurdo visto que $\sum_{i} \alpha_{i}=1$. Portanto, 


$$
f_{1}\left(\lambda_{\mu}, \lambda_{1}\right)=h\left(\lambda_{\mu}, \lambda_{1}\right)^{\prime} \sum^{-1} h\left(\lambda_{\mu}, \lambda_{1}\right)=\chi_{1 \%}
$$

Substituindo (9) na restrição de (P2) obtemos

$$
\begin{gathered}
-\lambda_{P 1} \cdot \sum_{i} g_{i}\left(\lambda_{\mu}, \lambda_{1}\right)=1, \\
-\lambda_{P 1} \cdot \sum_{i} g_{i}\left(\lambda_{\mu}, \lambda_{1}\right) \cdot\left(r+S_{i}-\left(1+r+S_{i}-R_{i}\right) \cdot \Phi\left(\bar{x}^{i}\right)\right)=\mu .
\end{gathered}
$$

Finalmente, dividindo (12) por (11) para cancelar $\lambda_{P 1}$, obtemos a equação

$$
\begin{aligned}
f_{2}\left(\lambda_{\mu}, \lambda_{1}\right) & \equiv \frac{\sum_{i} g_{i}\left(\lambda_{\mu}, \lambda_{1}\right) \cdot\left(r+S_{i}-\left(1+r+S_{i}-R_{i}\right) \cdot \Phi\left(\bar{x}^{i}\right)\right)}{\sum_{i} g_{i}\left(\lambda_{\mu}, \lambda_{1}\right)} \\
& =\mu .
\end{aligned}
$$

Os valores $\lambda_{\mu}$ e $\lambda_{1}$ são obtidos resolvendo o sistema $2 \times 2$ de equações não lineares (10') e (13). Daí a equação (11) nos fornece $\lambda_{P 1}$. Das equações (8) e (9) obtemos as soluções de (P1') e (P2) ${ }^{4}$. Conseqëntemente,

Independentemente do número de ativos ou produtos bancários, o problema numérico é reduzido a solucionar um sistema de 2 variáveis e 2 equações não lineares.

O gráfico 2 mostra um exemplo numérico. Grafando os valores Risk $(\mu)$ contra $\mu$ sugere que a fronteira ótima da nossa otimização tem formato semelhante ao da fronteira ótima do programa clássico de média-variância de Markowitz. De fato, pode-se demonstrar que a fronteira ótima da nossa otimização compartilha de várias propriedades obtidas no modelo de Markowitz:

Proposição 1:

i) A função $\operatorname{Risco}(\mu)$ é convexa.

ii) Existe um portfólio de risco mínimo e um nível de retorno esperado associado $\bar{\mu}$ tal que $\frac{\partial \operatorname{Risco}(\mu)}{\partial \mu} \geq 0 \Leftrightarrow . \mu \geq \bar{\mu}$

\footnotetext{
${ }^{4}$ Como RT não é convexa em $z$, as C.P.O. de (P1') não são, a princípio, necessárias e suficientes para caracterizar o ótimo. O argumento do texto ainda assim funciona porque a função RT é linear em $\alpha$ e, por aplicação do Teorema do Envelope, a equação (8) fornece um único valor de $z^{i}$.
} 
iii) O gráfico Risco $(\mu)$ tem assíntotas quando $\mu \rightarrow \pm \infty$.

Gráfico 2 - Exemplo numérico.

\begin{tabular}{|c|c|c|}
\hline $\begin{array}{ll}S_{1}=20 \%, & R_{1}=50 \%, \\
S_{2}=25 \%, & R_{2}=50 \%, \\
S_{3}=25 \%, & R_{3}=20 \%, \\
S_{4}=30 \%, & R_{4}=30 \%, \\
S_{5}=18 \%, & R_{5}=40 \%,\end{array}$ & $\begin{array}{l}P_{d}^{1}=\Psi\left(\bar{x}^{1}\right)=8 \%, \\
P_{d}^{2}=\Psi\left(\bar{x}^{2}\right)=10 \%, \\
P_{d}^{3}=\Psi\left(\bar{x}^{3}\right)=10 \%, \\
P_{d}^{4}=\Psi\left(\bar{x}^{4}\right)=12 \%, \\
P_{d}^{5}=\Psi\left(\bar{x}^{5}\right)=10 \%,\end{array}$ & $\begin{array}{l}\text { Ret. } E s p_{1}=32.8 \% \\
\text { Ret. } E s p_{2}=35.5 \% \\
\text { Ret. } E s p_{3}=32.5 \% \\
\text { Ret. } E s p_{4}=35.6 \% \\
\text { Ret.Esp }=28.2 \%\end{array}$ \\
\hline$\rho_{1}=0.9, \quad \rho_{2}=0.8$ & $\rho_{3}=0.6, \rho_{4}=0.5$ & $\rho_{5}=0.45$ \\
\hline
\end{tabular}

$$
\sum=\left[\begin{array}{ccccc}
1 & 0.2 & 0.3 & 0.3 & 0.3 \\
0.2 & 1 & 0.5 & 0.4 & 0.3 \\
0.3 & 0.5 & 1 & 0.2 & 0.2 \\
0.3 & 0.4 & 0.2 & 1 & 0.15 \\
0.3 & 0.3 & 0.2 & 0.15 & 1
\end{array}\right] r=20 \%
$$

Porém, nossos portfólios ótimos não compartilham de uma importante propriedade do modelo de Markowitz. Como é bem sabido, o conjunto de portfólios ótimos de média-variância de Markowitz é fechado para combinações lineares. Esta propriedade não é obtida aqui. Os exemplos numéricos do gráfico 2 nos fornecem os seguintes portfólios ótimos $\alpha^{*}$

$$
\begin{gathered}
\mu=32 \% \rightarrow \alpha^{*}(32 \%)=[10.6 \%, 18.8 \%, 7.5 \%, 21.9 \%, 41.2 \%] \\
\mu=33 \% \rightarrow \alpha^{*}(33 \%)=[9.2 \%, 38.2 \%, 5.4 \%, 18.3 \%, 28.9 \%] \\
\mu=32.5 \% \rightarrow \alpha^{*}(32.5 \%)=[10.2 \%, 27.8 \%, 6.5 \%, 18.3 \%, 28.9 \%]
\end{gathered}
$$

É fácil ver que o portfólio $\frac{1}{2} \alpha^{*}(32 \%)+\frac{1}{2} \alpha^{*}(33 \%)$ tem retorno esperado $32.5 \%$. Porém, o portfólio ótimo para este retorno esperado é $\alpha^{*}(32.5 \%)$, que é diferente do portfólio $\frac{1}{2} \alpha^{*}(32 \%)+\frac{1}{2} \alpha^{*}(33 \%)$. Portanto, esta combinação linear não é um portfólio sub-ótimo e não está na fronteira eficiente.

Recordando resultados bem conhecidos do modelo de Markowitz - ver Huang e Litzemberg (1988) - lembramos que esta importante propriedade (combinações lineares de portfólio ótimo são portfólios ótimos) implica 1) o portfólio de mercado está na fronteira ótima e 2) CAPM. Estas duas importantes implicações são as mais freqüentemente utilizadas para testar empiricamente o modelo de Markowitz. 
Contudo, ao contrário dos portfólios ótimos de (P2), introduzindo um ativo sem risco na otimização restaura esta importante propriedade e abre caminho para possíveis testes econométricos.

\section{Ativo sem risco.}

Se incluirmos um ativo sem risco com retorno $r$, o programa (P2) se torna

$\operatorname{Risco}^{r}(\mu)=\min _{\alpha}-P M^{r}(\alpha)$

$$
\text { s.t. } \sum_{i} \alpha_{i}\left(r+S_{i}-\left(1+r+S_{i}-R_{i}\right) \Phi\left(\bar{x}^{i}\right)\right)+\left(1-\sum_{i} \alpha_{i}\right) . r=\mu
$$

onde $P M^{r}(\alpha)$ é a solução de $\left(P 1^{\prime}\right)$ com $R T(\alpha, Z)$ substituído por $R T^{r}(\alpha, Z)+\left(1-\sum_{i} \alpha_{i}\right) \cdot r$.

Outro resultado importante obtido no ambiente de Markowitz é replicado aqui.

Proposição 2: O gráfico $\operatorname{Risco}^{r}(\mu)$ é uma reta tangente a $\operatorname{Risco}(\mu)$ e o portfólio ótimo é uma combinação linear do portfólio tangente e o ativo sem risco.

Portanto, diferente do problema $(P 2)$, a combinação linear de portfólios ótimos de $\left(P 2^{\prime}\right)$ é também um portfólio ótimo. Portanto, o portfólio de mercado está na fronteira ótima. Utilizaremos este fato para desenvolver implicações que podem ser testadas econometricamente.

\section{Considerações Econométricas.}

Seja $\tilde{r}_{j}$ o retorno do ativo $j$ e $\tilde{r}_{M}$ o retorno do portfólio de mercado. O modelo de média-variância de Markowitz tem CAPM como a mais importante implicação do ponto de vista econométrico. Na sua forma mais simples CAPM-Markowitz afirma que

$$
E\left(\tilde{r}_{j}\right)-r=\beta_{j M}\left[E\left(\tilde{r}_{M}\right)-r\right],
$$

onde $\beta_{j M}=\beta_{j M}^{M a r k o w i t z}=\frac{\operatorname{Cov}\left(\tilde{r}_{j}, \tilde{r}_{M}\right)}{\operatorname{VAR}\left(\tilde{r}_{M}\right)}$ é o "beta" do ativo $j$, cuja forma funcional é específica do modelo de Markowitz e suas hipóteses - com destaque para a hipótese de que os ativos têm distribuição normal.

O modelo CAPM, sintetizado na fórmula (14), é também obtido através de condições de equilíbrio de economias com agente representativo (ver 
Duffie (2001)). Neste caso a forma funcional de "beta" é $\beta_{j M}^{\text {Equilibrio }}=$ $\frac{\operatorname{Cov}\left(\tilde{r}_{j}, u^{\prime}(\tilde{M})\right)}{\operatorname{Cov}\left(\tilde{r}_{M}, u^{\prime}(\tilde{M})\right)}$, onde $\tilde{M}$ é a riqueza agregada do mercado e $u^{\prime}($.$) a utilidade$ marginal do agente representativo. Sob as hipóteses de distribuição normal dos ativos e de a riqueza agregada ser igual ao portfólio de mercado, é possível reconciliar $\beta_{j M}^{\text {Equilibrio }}=\beta_{j M}^{\text {Markowitz }}$.

Nosso modelo de otimização de risco de crédito agregado também tem como implicação a equação (14). E, de forma análoga, nossa versão do CAPM possui uma forma funcional específica para o "beta".

Seja $\alpha^{M}=\left[\alpha_{1}^{M}, \alpha_{2}^{M}, \ldots, \alpha_{N}^{M}\right]$ os pesos do portfólio de mercado. Como este portfólio é um elemento da fronteira eficiente, ele é solução da otimização $\left(P 2^{\prime}\right)$ com $\mu=E\left(\tilde{r}_{M}\right)$. Além disso, após otimizada, a função objetivo nos fornece $\operatorname{Risco}\left(\tilde{r}_{M}\right) \equiv \operatorname{Risco}\left(E\left(\tilde{r}_{M}\right)\right)=-P M\left(\alpha^{M}\right)=$ $-R T\left(\alpha^{M}, z^{*}\right)$, onde $z^{*}=\left[z_{1}^{*}, z_{2}^{*}, \ldots, z_{N}^{*}\right]$ é solução de $\left(P 1^{\prime}\right)$ e pode ser interpretado como choque macroeconômico desfavorável o suficiente - $1 \%$ de probabilidade - para gerarem a perda de valor $\operatorname{Risco}\left(E\left(\tilde{r}_{M}\right)\right)$ no portfólio de mercado.

Defina o risco marginal do ativo $j$ no portfólio de mercado como a contribuição deste ativo para a perda $\operatorname{Risco}\left(E\left(\tilde{r}_{M}\right)\right)$. Matematicamente temos a notação

$$
R i s c o_{-} M \arg \left(\tilde{r}_{j}, \tilde{r}_{M}\right)=S_{j}-\left(1+r+S_{j}-R_{j}\right) \Phi\left(\frac{\bar{x}^{j}-\sqrt{\rho_{j}} z_{j}^{*}}{\sqrt{1-\rho_{j}}}\right)
$$

Finalmente, defina $\beta_{j M}^{C r e d i t o}=\frac{\operatorname{Risco}{ }_{j} M \arg \left(\tilde{r}_{j}, \tilde{r}_{M}\right)}{\operatorname{Risco}\left(\tilde{r}_{M}\right)}$. Assim, temos o resultado

Teorema 3:

A otimização $\left(P 2^{\prime}\right)$ implica $E\left(\tilde{r}_{j}\right)-r=\beta_{j M}^{C r e d i t o}\left[E\left(\tilde{r}_{M}\right)-r\right]$, versão do modelo CAPM para ativos de crédito.

Supondo que tenhamos uma série histórica de retornos, um possível teste econométrico do modelo consiste em computar $\operatorname{Risco}\left(\tilde{r}_{M}\right)$ como o quantil $1 \%$ dos retornos do portfólio de mercado e Risco_ $M \arg \left(\bar{r}_{j}, \bar{r}_{M}\right)$ a contribuição do ativo $j$ para este risco. Desta forma, o "beta" $\beta_{j M}^{\text {Credito }}$ de cada ativo pode ser calculado. Depois, estimar $E\left(\tilde{r}_{j}\right)$ como a média da série temporal dos retornos do ativo $j$. Por fim, proceder com a regressão de $E\left(\tilde{r}_{j}\right)-r$ sobre os "betas". Isto é,

$$
E\left(\tilde{r}_{j}\right)-r=a+b \cdot \beta_{j M}^{C r e d i t o}+\tilde{\varepsilon}_{j}, \quad j=1,2, \ldots, N
$$


Como implicação do Teorema 3 temos $a=0$. Além disso, é óbvio que o portfólio de mercado tem que satisfazer $E\left(\tilde{r}_{M}\right)-r \geq 0$, pois ele é um portfólio ótimo e o ativo sem risco está entre as possíveis escolhas. Conseqüentemente temos para testar a hipótese nula $H 0: a=0$ e $b \geq 0$.

Por falta de banco de dados de qualidade, neste trabalho não procedemos com o efetivo teste econométrico descrito acima. Além disso, o teste econométrico proposto acima é por demais simplificado e passível dos mesmos problemas conceituais e dificuldades econométricas associados aos testes de CAPM.

Por exemplo, introduzimos erro amostral ao utilizar a série temporal de $\tilde{r}_{M}$ para computar o quantil $1 \%$ de $\operatorname{Risco}\left(\tilde{r}_{M}\right)$. Portanto, as estimativas de $\beta_{j M}^{C r e d i t o}$ conterão erro amostral e, consequentemente, os estimadores $\hat{a}$ e $\hat{b}$ da regressão (15) serão viesados, ineficientes e podem até ser inconsistentes.

No contexto de Markowitz este problema foi superado substituindo a regressão ordinária de mínimos quadrados por regressões de dois estágios ver Shanken (1992) - ou por regresssões GMM - ver Mackinlay e Richardson (1991). A incerteza "adicional" do erro amostral em $\beta_{j M}^{\text {Markowitz induz }}$ uma correção na distribuição assintótica destes estimadores. Estes autores obtêm os ajustes necessários (em relação à distribuição assintótica OLS) na variância da distribuição limite.

Estas estratégias certamente funcionariam neste nosso contexto de portfólios de risco de crédito. Porém, por causa das diferenças nas formas funcionais de $\beta_{j M}^{M a r k o w i t z}$ e $\beta_{j M}^{C r e d i t o}$, é provável que os ajustes na variância assintótica sejam diferentes dos obtidos por estes autores. Os detalhamentos desta análise assintótica são postergados para uma eventual extensão do trabalho.

Outros problemas econométricos potenciais são: não estacionariedade das séries de "betas" e prêmios de risco, falta de uma boa aproximação para o portfólio de mercado, heterocedasticidade, etc. No contexto de CAPM tradicional estas dificuldades foram enfrentadas em Blume e Friend (1973), Fama e MacBeth $(1972$, 1973) e Black, Jensen e Scholes (1972), além de outros. De fato, acreditamos que boa parte da literatura desenvolvida para aperfeiçoar os testes empíricos de CAPM tradicional pode ser adaptada para o contexto deste trabalho de portfólios de risco de crédito.

\section{Conclusão.}

Neste trabalho abordamos o risco de uma carteira de crédito pulverizado. Na literatura, existem outros trabalhos que abordam o risco de crédito de forma agregada, por exemplo CreditMetrics do JP Morgan e 
CreditRisk+ do CSFB. Porém, estes modelos têm limitações quando aplicados a alguns produtos bancários, especialmente em mercados emergentes onde a taxa de inadimplência é alta. O modelo desenvolvido neste artigo supera estas limitações.

Utilizando o conceito de risco de Perda Máxima, alternativo ao conceito mais tradicional de Valor a Risco, elaboramos um programa de otimização de portfólios que é analiticamente tratável. Demonstramos que os portfólios ótimos obtidos na nossa otimização têm propriedades qualitativas semelhantes aos portfólios ótimos de Markowitz. Obtemos também um modelo de CAPM para portfólios de créditos. Este resultado abre caminho para futuras extensões e aplicações econométricas deste trabalho.

Submetido em Agosto de 2003. Revisado em Novembro de 2003. 


\section{Referências}

Black, F., Jensen, M. \& Scholes, M. 1972. "The Capital Asset Pricing Model: Some empirical tests". Em Studies in the Theory of Capital Markets, Edited by M. Jensen. Praeger, New York.

Blume, M. \& Friend, I. 1973. "A New Look at the Capital Asset Pricing Model". Journal of Finance, 28: 19-33.

Duffie, D. 2001. "Dynamic Asset Pricing Theory", Princeton University Press.

Fama, E. \& MacBeth, J. 1973. "Risk, Return and Equilibrium: Empirical Tests". Journal of Political Economy, 81: 607-636.

Huang C. F. \& Litzemberg, R. H. 1988. "Foundations for Financial Economics".

Mackinlay, A. \& Richardson, M. 1991. "Using Generalized Methods of Moments to Test Mean Variance Efficiency". Journal of Finance, 46: $511-527$.

Shanken, J. 1992. "On the estimation of Beta-Pricing Models". Review of Financial Studies, 5: 1-33.

Shiryaev, A.N. 1996. Probability. New York: Springer Verlag.

Studer, G. 1995. "Value at Risk and Maximum Loss Optimization", Risklab Technical Report. 


\section{Apêndice I. Demonstrações.}

\section{Teorema 1:}

Defina $Y_{n}=\frac{\chi_{\left[X_{1} \leq \bar{x}\right]}+\chi_{\left[X_{2} \leq \bar{x}\right]}+\chi_{\left[X_{n} \leq \bar{x}\right]}}{n}$, onde $\chi_{\left[X_{i} \leq \bar{x}\right]}=\left\{\begin{array}{ll}1 & \text { se } X_{i} \leq \bar{x} \\ 0 & \text { se } X_{i}>\bar{x}\end{array}\right.$, onde as variáveis aleatórias $X_{i}$ estão definidas no texto. Então $Y_{n} \stackrel{D}{\rightarrow} \Phi\left(\frac{\bar{x}-\sqrt{\rho} Z}{\sqrt{1-\rho}}\right)$.

Demonstração: Condicionando em $Z=z, Y_{n} \mid Z=z$ é a soma de variáveis aleatórias independentes de Bernoulli com parâmetro $p_{z}=P\left(X_{1} \leq \bar{x}\right)=$ $\Phi\left(\frac{\bar{x}-\sqrt{\rho} z}{\sqrt{1-\rho}}\right)$. Pela Lei dos grandes números (Shiryaev [1996] - página 325)

$$
Y_{n} \mid Z=z \stackrel{P}{\rightarrow} p_{z}
$$

Fixado $z$, por (AI.1) a função característica condicionada $E\left(e^{i u Y_{n}} \mid Z=z\right)$ converge para:

$$
E\left(e^{i u Y_{n}} \mid Z=z\right) \rightarrow e^{i u p_{z}}, \forall u \in \mathcal{R}
$$

Fixe $u \in \mathcal{R}$ arbitrário. Note que como funções de $z,\left\{E\left(e^{i u Y_{n}} \mid Z=z\right)\right\}_{n>1}$ são limitadas pela função constante $f(z)=1, \forall z \in \mathcal{R}$ e por (AI.2) converge pontualmente (para cada $z$ ) para $e^{\text {iup } z}$. Portanto, pelo Teorema da Convergência Dominada, a seqüencia de suas expectativas $\left\{E\left(e^{i u Y_{n}}\right)\right\}_{n \geq 1}$ converge para a expectativa da v.a. $e^{i u p_{Z}}$, que é exatamente a função característica da v.a. $\Phi\left(\frac{\bar{x}-\sqrt{\rho} z}{\sqrt{1-\rho}}\right)$ no ponto $u$. Assim, as funções características das v.a. $Y_{n}$ convergem para a função característica de $\Phi\left(\frac{\bar{x}-\sqrt{\rho} z}{\sqrt{1-\rho}}\right)$, ou em outras palavras, converge em distribuição.

\section{Teorema 2:}

$Y_{n} \stackrel{P}{\rightarrow} Y \equiv \Phi\left(\frac{\bar{x}-\sqrt{\rho} Z}{\sqrt{1-\rho}}\right)$ com uma taxa de convergência exponencial.

Demonstração: Condicionando em $Z=z, Y_{n} \mid Z=z$ é a soma de variáveis aleatórias independentes de Bernoulli com parâmetro $p_{z}=P\left(X_{1} \leq \bar{x}\right)=$ $\Phi\left(\frac{\bar{x}-\sqrt{\rho} z}{\sqrt{1-\rho}}\right)$. Utilizando um resultado conhecido na Lei dos Grandes Números relacionado a velocidade de convergência de v.a. independentes de Bernoulli (Shiryaev [1996] - página 70), para qualquer $\varepsilon>0$

$$
P\left(\left|Y_{n}-Y\right| \geq \varepsilon \mid Z=z\right) \leq 2 e^{-2 n \varepsilon^{2}}
$$


Note que o limite superior em (AI.3) não depende do valor específico condicionado de $z$. Integrando em $Z$ e usando (AI.3) tem-se:

$$
P\left(\left|Y_{n}-Y\right| \geq \varepsilon\right)=\int_{\mathcal{R}} P\left(\left|Y_{n}-Y\right| \geq \varepsilon \mid Z=z\right) P_{z}(d z) \leq 2 e^{-2 n \varepsilon^{2}}
$$

onde $P_{Z}$ denota a distribuição de probabilidade da v.a. $Z$.

\section{lema.}

A função $P M(\alpha)$ é côncava.

\section{Demonstração:}

Fixados os parâmetros $\alpha_{1}, \alpha_{2}$ e $\left(\lambda . \alpha_{1}+(1-\lambda) . \alpha_{2}\right)$, sejam $z_{1}, z_{2}$ e $z_{\lambda}$ as respectivas soluções do programa (P1'). Assim, $P M\left(\alpha_{1}\right)=R T\left(\alpha_{1}, z_{1}\right) \leq$ $R T\left(\alpha_{1}, z_{\lambda}\right), P M\left(\alpha_{2}\right)=R T\left(\alpha_{2}, z_{2}\right) \leq R T\left(\alpha_{2}, z_{\lambda}\right)$ e $P M\left(\lambda \alpha_{1}+(1-\lambda) \alpha_{2}\right)$ $=R T\left(\lambda \alpha_{1}+(1-\lambda) \alpha_{2}, z_{\lambda}\right)$.

Porém, $R T(\alpha, z)$ é uma função linear em $\alpha$. Conseqüentemente, $P M\left(\lambda \alpha_{1}+(1-\lambda) \alpha_{2}\right)=\lambda \cdot R T\left(\alpha_{1}, z_{\lambda}\right)+(1-\lambda) \cdot R T\left(\alpha_{2}, z_{\lambda}\right) \geq \lambda \cdot P M\left(\alpha_{1}\right)+$ $(1-\lambda) \cdot P M\left(\alpha_{2}\right)$.

\section{Proposição 1 (i)}

A função $\operatorname{Risk}(\mu)$ é convexa.

Demonstração: Fixados os parâmetros $\mu_{1}, \mu_{2}$ e $\left(\lambda . \mu_{1}+(1-\lambda) \cdot \mu_{2}\right)$, sejam $\alpha_{1}, \alpha_{2}$ e $\alpha_{\lambda}$ as respectivas soluções do programa (P2). Assim, Risk $\left(\mu_{1}\right)=$ $-P M\left(\alpha_{1}\right), \operatorname{Risk}\left(\mu_{2}\right)=-P M\left(\alpha_{2}\right.$ e $\operatorname{Risk}\left(\lambda \mu_{1}+(1-\lambda) \mu_{2}\right)=-P M\left(\alpha_{\lambda}\right)$.

Observe que $\left(\lambda . \alpha_{1}+(1-\lambda) \cdot \alpha_{2}\right)$ satisfaz as restrições do programa (P2) com parâmetro $\left(\lambda \cdot \mu_{1}+(1-\lambda) \cdot \mu_{2}\right)$. Conseqüentemente,

$$
\operatorname{Risk}\left(\lambda \mu_{1}+(1-\lambda) \mu_{2}\right) \leq-P M\left(\lambda \alpha_{1}+(1-\lambda) \alpha_{2}\right)
$$

Pelo lema 3, $P M($.$) é côncava e, portanto,$

$$
P M\left(\lambda \alpha_{1}+(1-\lambda) \alpha_{2}\right) \geq \lambda \cdot P M\left(\alpha_{1}\right)+(1-\lambda) \cdot P M\left(\alpha_{2}\right)
$$

Logo,

$\operatorname{Risk}\left(\lambda \mu_{1}+(1-\lambda) \mu_{2}\right) \leq-\lambda \cdot P M\left(\alpha_{1}\right)-(1-\lambda) \cdot P M\left(\alpha_{2}\right)=\lambda \cdot \operatorname{Risk}\left(\mu_{1}\right)+$ $(1-\lambda) \cdot P M\left(\mu_{2}\right)$.

\section{Proposição 1 (ii)}


Existe um portfólio de risco mínimo e um nível de retorno esperado associado tal que

$$
\frac{\partial \operatorname{Risco}(\mu)}{\partial \mu} \geq 0 \Leftrightarrow \mu \geq \bar{\mu} .
$$

Demonstração: Seja $\alpha^{*}(\mu)$ a solução do programa $(\mathrm{P} 2)$. Assim, $\operatorname{Risco}(\mu)=$ $-P M\left(\alpha^{*}(\mu)\right)$ e, portanto,

$$
\frac{\partial \operatorname{Risco}(\mu)}{\partial \mu}=-\sum_{i} P M_{\alpha_{i}} \cdot \frac{\partial \alpha_{i}^{*}(\mu)}{\partial \mu} .
$$

Porém, pelas C.P.O. de (P2), $P M_{\alpha_{i}}=\lambda_{\mu}\left(r+S_{i}-\left(1+r+S_{i}-\right.\right.$ $\left.\left.R_{i}\right) . \Phi\left(\bar{x}^{i}\right)\right)+\lambda_{1}$. Denote por $v_{i}=r+S_{i}-\left(1+r+S_{i}-R_{i}\right) . \Phi\left(\bar{x}^{i}\right)$ o retorno esperado da atividade bancária $i$. Assim,

$$
\frac{\partial \operatorname{Risco}(\mu)}{\partial \mu}=-\lambda_{\mu} \sum_{i} v_{i} \frac{\partial \alpha_{i}^{*}(\mu)}{\partial \mu}-\lambda_{1} \frac{\partial \alpha_{i}^{*}(\mu)}{\partial \mu}=-\lambda_{\mu}
$$

onde a última igualdade é obtida derivando as restrições de (P2) em relação a $\mu$.

Conforme desenvolvido no texto, $\left(\lambda_{\mu}, \lambda_{1}\right)$ é a solução do sistema de equações (10) e (13).

$$
\left\{\begin{array}{l}
h\left(\lambda_{\mu}, \lambda_{1}\right)^{\prime} \sum^{-1} h\left(\lambda_{\mu}, \lambda_{1}\right)=\chi_{1 \%} \\
\sum_{i} \alpha_{i} \cdot v_{i}=\sum_{i}\left(\frac{g_{i}\left(\lambda_{\mu}, \lambda_{1}\right)}{\sum_{j} g_{j}\left(\lambda_{\mu}, \lambda_{1}\right)}\right) \cdot v_{i}=\mu
\end{array}\right.
$$

Derivando em relação a $\mu$. obtemos

$$
\left\{\begin{array}{l}
{\left[\left(\frac{\partial h}{\partial \lambda_{\mu}}\right)^{\prime} \sum^{-1} h\left(\lambda_{\mu}, \lambda_{1}\right)\right] \cdot \frac{\partial \lambda_{\mu}}{\partial \mu}+\left[\left(\frac{\partial h}{\partial \lambda_{1}}\right)^{\prime} \sum^{-1} h\left(\lambda_{\mu}, \lambda_{1}\right)\right] \cdot \frac{\partial \lambda_{1}}{\partial \mu}=0} \\
{\left[\sum_{i} \frac{\partial \alpha_{i}^{*}}{\partial \lambda_{\mu}} \cdot v_{i}\right] \frac{\partial \lambda_{\mu}}{\partial \mu}+\left[\sum_{i} \frac{\partial \alpha_{i}^{*}}{\partial \lambda_{1}} \cdot v_{i}\right] \frac{\partial \lambda_{\mu}}{\partial \mu}=1}
\end{array}\right.
$$

Analisemos a primeira equação deste sistema. As derivadas parciais em relação a $\lambda_{\mu}$ e $\lambda_{1}$ da equação (8) nos fornecem

$$
\frac{\partial h_{i}}{\partial \lambda_{1}}=\frac{1}{\left(1+r+S_{i}-R_{i}\right) \sqrt{\frac{\rho_{i}}{1-\rho_{i}}} \phi\left(\frac{\bar{x}-\sqrt{\rho_{i}} h_{i}\left(\lambda_{\mu}, \lambda_{1}\right)}{\sqrt{1-\rho_{i}}}\right)}
$$


$\mathrm{e}$

$$
\frac{\partial h_{i}}{\partial \lambda_{\mu}}=v_{i} \frac{\partial h}{\partial \lambda_{1}} \rightarrow \frac{\partial h}{\partial \lambda_{\mu}}=V \cdot \frac{\partial h}{\partial \lambda_{1}}
$$

$V$ é a matriz diagonal de componentes $v_{i}$.

Conseqüentemente, a equação (9) pode ser reescrita como $g_{i}=$ $\left(\frac{\partial h_{i}}{\partial \lambda_{1}} \cdot e_{i}\right)^{\prime} \sum^{-1} h$, onde $e_{i}$ é o vetor $n$ dimensional com 1 no $i$-ésimo componente e zero nos outros. Assim,

$$
\left(\frac{\partial h}{\partial \lambda_{1}}\right)^{\prime} \sum^{-1} h=\sum_{i}\left[\left(\frac{\partial h_{i}}{\partial \lambda_{1}} \cdot e_{i}\right)^{\prime} \sum^{-1} h\right]=\sum_{i} g_{i}
$$

multiplicando $g_{i}$ por $v_{i}$ e somando temos

$$
\begin{aligned}
\sum_{i} v_{i} . g_{i} & =\sum_{i}\left[\left(v_{i} \frac{\partial h_{i}}{\partial \lambda_{1}} \cdot e_{i}\right)^{\prime} \sum^{-1} h\right] \\
& =\sum_{i}\left[\left(\frac{\partial h_{i}}{\partial \lambda_{\mu}} \cdot e_{i}\right)^{\prime} \sum^{-1} h\right]=\left(\frac{\partial h}{\partial \lambda_{\mu}}\right)^{\prime} \sum^{-1} h
\end{aligned}
$$

assim, a primeira equação em (AI.7) se transforma em

$$
\begin{aligned}
\frac{\partial \lambda_{1}}{\partial \mu} & =-\frac{\left[\left(\frac{\partial h}{\partial \lambda_{\mu}}\right)^{\prime} \sum^{-1} h\left(\lambda_{\mu}, \lambda_{1}\right)\right]}{\left[\left(\frac{\partial h}{\partial \lambda_{1}}\right)^{\prime} \sum^{-1} h\left(\lambda_{\mu}, \lambda_{1}\right)\right]} \cdot \frac{\partial \lambda_{\mu}}{\partial \mu} \\
& =-\frac{\sum_{i} v_{i} g_{i}}{\sum_{i} g_{i}} \cdot \frac{\partial \lambda_{\mu}}{\partial \mu}=-\sum_{i}\left[v_{i} \frac{g_{i}}{\sum_{i} g_{i}}\right] \cdot \frac{\partial \lambda_{\mu}}{\partial \mu} \\
& =-\sum_{i}\left[v_{i} \cdot \alpha_{i}^{*}\right] \cdot \frac{\partial \lambda_{\mu}}{\partial_{\mu}}=-\mu \cdot \frac{\partial \lambda_{\mu}}{\partial \mu}
\end{aligned}
$$

substituindo na segunda equação de (AI.7) obtemos

$$
\begin{aligned}
1 & =\left[\sum_{i} \frac{\partial \alpha_{i}^{*}}{\partial \lambda_{\mu}} \cdot v_{i}\right] \frac{\partial \lambda_{\mu}}{\partial \mu}+\left[\sum_{i} \frac{\partial \alpha_{i}^{*}}{\partial \lambda_{1}} \cdot v_{i}\right] \frac{\partial \lambda_{1}}{\partial \mu} \\
& =\left\{\left[\sum_{i} \frac{\partial \alpha_{i}^{*}}{\partial \lambda_{\mu}} \cdot v_{i}\right]-\mu \cdot\left[\sum_{i} \frac{\partial \alpha_{i}^{*}}{\partial \lambda_{1}} \cdot v_{i}\right]\right\} \frac{\partial \lambda_{\mu}}{\partial \mu}
\end{aligned}
$$


Porém, um pouco de álgebra nos dá

$$
\begin{aligned}
\sum_{i} \frac{\partial \alpha_{i}^{*}}{\partial \lambda_{\mu}} \cdot v_{i} & =\frac{\sum_{i}\left[\frac{\partial g_{i}}{\partial \lambda_{\mu}}-\left(\sum_{i} \frac{\partial g_{i}}{\partial \lambda_{\mu}}\right) \cdot\left(\frac{g_{i}}{\sum_{i} g_{i}}\right)\right] \cdot v i}{\sum_{i} g_{i}} \\
& =\frac{\sum_{i} \frac{\partial g_{i}}{\partial \lambda_{\mu}} \cdot v_{i}-\left(\sum_{i} \frac{\partial g_{i}}{\partial \lambda_{\mu}}\right) \sum_{i} \alpha_{i}^{*} \cdot v_{i}}{\sum_{i} g_{i}} \\
& =\frac{\sum_{i} \frac{\partial g_{i}}{\partial \lambda_{\mu}} v_{i}-\left(\sum_{i} \frac{\partial g_{i}}{\partial \lambda_{\mu}}\right) \mu}{\sum_{i} g_{i}}=\frac{\sum_{i} \frac{\partial g_{i}}{\partial \lambda_{\mu}}\left(v_{i}-\mu\right)}{\sum_{i} g_{i}}
\end{aligned}
$$

e, de forma semelhante, obtemos $\sum_{i} \frac{\partial \alpha_{i}}{\partial \lambda_{1}} \cdot v_{i}=\frac{\sum_{i} \frac{\partial g_{i}}{\partial \lambda_{1}}\left(v_{i}-\mu\right)}{\sum_{i} g_{i}}$

Com isso, a expressão dentro dos colchetes em (AI.9) pode ser reescrita como

$$
\left[\sum_{i} \frac{\partial \alpha_{i}^{*}}{\partial \lambda_{\mu}} \cdot v_{i}\right]-\mu \cdot\left[\sum_{i} \frac{\partial \alpha_{i}^{*}}{\partial \lambda_{1}} \cdot v_{i}\right]=\frac{\sum_{i}\left(\frac{\partial g_{i}}{\partial \lambda_{\mu}}-\mu \frac{\partial g_{i}}{\partial \lambda_{1}}\right)\left(v_{i}-\mu\right)}{\sum_{i} g_{i}}
$$

porém

$$
\begin{aligned}
\frac{\partial g_{i}}{\partial \lambda_{\mu}}-\mu \frac{\partial g_{i}}{\partial \lambda_{1}} & =\left(\frac{\partial h_{i}}{\partial \lambda_{1}} \cdot e_{i}\right)^{\prime} \sum^{-1}\left(\frac{\partial h}{\partial \lambda_{\mu}}-\mu \frac{\partial h}{\partial \lambda_{1}}\right) \\
& =\left(\frac{\partial h_{i}}{\partial \lambda_{1}} \cdot e_{i}\right)^{\prime} \sum^{-1}\left(V \frac{\partial h}{\partial \lambda_{1}}-\mu \frac{\partial h}{\partial \lambda_{1}}\right) \\
& =\left(\frac{\partial h_{i}}{\partial \lambda_{1}} \cdot e_{i}\right)^{\prime} \sum^{-1}(V-\mu . I) \frac{\partial h}{\partial \lambda_{1}}
\end{aligned}
$$

$\log \mathrm{O}$

$$
\begin{aligned}
& \frac{\sum_{i}\left(\frac{\partial g_{i}}{\partial \lambda_{\mu}}-\mu \frac{\partial g_{i}}{\partial \lambda_{1}}\right)\left(v_{i}-\mu\right)}{\sum_{i} g_{i}} \\
= & \frac{\sum_{i}\left(\left(v_{i}-\mu\right) \frac{\partial h_{i}}{\partial \lambda_{1}} \cdot e_{i}\right)^{\prime} \sum^{-1}(V-\mu . I) \frac{\partial h}{\partial \lambda_{1}}}{\sum_{i} g_{i}} \\
= & \frac{\frac{\partial h}{\partial \lambda_{1}}(V-\mu \cdot I)^{\prime} \sum^{-1}(V-\mu . I) \frac{\partial h}{\partial \lambda_{1}}}{\sum_{i} g_{i}}
\end{aligned}
$$


A matriz de variância-covariância $\sum$ é positiva definida. Logo a inversa $\sum^{-1}$ também é e, portanto, $\frac{\partial h}{\partial \lambda_{1}}(V-\mu . I)^{\prime} \sum^{-1}(V-\mu . I) \frac{\partial h}{\partial \lambda_{1}}>0$. Além disso, o multiplicador $\lambda_{p 1}$ é positivo o que implica $\sum_{i} g_{i}<0$ pela equação (11). Conseqüentemente, $\left[\sum_{i} \frac{\partial \alpha_{i}^{*}}{\partial \lambda_{\mu}} \cdot v_{i}\right]-\mu \cdot\left[\sum_{i} \frac{\partial \alpha_{i}^{*}}{\partial \lambda_{1}} \cdot v_{i}\right]<0$ e, pela equação (AI.9), temos $\frac{\partial \lambda_{\mu}}{\partial \mu}>0$. Observando (AI.6) concluímos que $\frac{\partial \operatorname{Risco}(\mu)}{\partial \mu}$ é uma função estritamente crescente. Definido $\bar{\mu}$ o retorno esperado cuja solução do sistema de equações (10) e (13) nos dá $\lambda_{\mu}=0$, temos o resultado desejado.

\section{Proposição 1 (iii)}

O gráfico Risco $(\mu)$ tem assíntotas quando $\mu \rightarrow \pm \infty$.

Demonstração: O argumento de $\Phi^{-1}($.$) na equação (8) é uma probabilidade$ logo tem que ser menor que 1 e maior que zero. Consequentemente para todo produto bancário $i$,

$$
0 \leq \frac{\left(r+S_{i}-\lambda_{\mu}\left(r+S_{i}-\left(1+r+S_{i}-R_{i}\right) \cdot \Phi\left(\bar{x}^{i}\right)\right)-\lambda_{1}\right)}{1+r+S_{i}-R_{i}} \leq 1
$$

ou $R_{i}-1 \leq \lambda_{\mu} \cdot v_{i}+\lambda_{1} \leq r+S_{i} \quad \forall i$, onde $v_{i}=r+S_{i}-\left(1+r+S_{i}-R_{i}\right) \cdot \Phi\left(\bar{x}^{i}\right)$

As inequações acima definem, no plano cartesiano $\lambda_{1} \times \lambda_{\mu}$, uma região convexa de pontos factíveis. Esta região está limitada pelas retas

$$
\left\{\begin{array}{l}
\lambda_{\mu} \cdot v_{i}+\lambda_{1}=r+S_{i} \\
\lambda_{\mu} \cdot v_{j}+\lambda_{1}=R_{j}-1
\end{array} \quad \forall i, j\right.
$$

Sem perda de generalidade, suponha que $v_{i} \leq v_{j}$ para $i<j$. Defina

$$
\lambda_{\mu}^{\max }=\min _{i, j} \frac{1+r+S_{j}-R_{i}}{v_{j}-v_{i}}=\frac{1+r+S_{j^{*}}-R_{i^{*}}}{v_{j^{*}}-v_{i^{*}}}
$$

A região de pontos factíveis implica $-\lambda_{\mu}^{\max } \leq \lambda_{\mu} \leq \lambda_{\mu}^{\max }$. Da demonstração do Proposição 1 temos $\frac{\partial \operatorname{Risco}(\mu)}{\partial \mu}=-\lambda_{\mu}$ e $\frac{\partial \operatorname{Risco}(\mu)}{\partial \mu}$ é uma função estritamente crescente. Portanto, $\frac{\partial R i s c o(\mu)}{\partial \mu}$ é monótona e limitada superiormente e inferiormente. Logo esta função tem limites quando $\mu \rightarrow \pm \infty$, o que demonstra o resultado.

\section{Proposição 2}


O gráfico $\operatorname{Risco}^{r}(\mu)$ é uma reta tangente a $\operatorname{Risco}(\mu)$ e o portfólio ótimo é uma combinação linear do portfólio tangente e o ativo sem risco.

Demonstração: Seja $\alpha^{r}(\mu)$ a solução do programa (P2'). Assim, $\operatorname{Risco}^{r}(\mu)=-P M\left(\alpha^{r}(\mu)\right)$ e, portanto, $\frac{\partial \operatorname{Risco}(\mu)}{\partial \mu}=-\sum_{i} P M_{\alpha_{i}}^{r} \cdot \frac{\partial \alpha_{i}^{r}(\mu)}{\partial \mu}$.

Pelas C.P.O. de (P2'), $P M_{\alpha_{i}}^{r}=\lambda_{\mu}^{r}\left(S_{i}-\left(1+r+S_{i}-R_{i}\right) . \Phi\left(\bar{x}^{i}\right)\right)$, onde $\lambda_{\mu}^{r}$ é o multiplicador de Lagrange da restrição em (P2'). Assim,

$$
\begin{aligned}
\frac{\partial \operatorname{Risco}^{r}(\mu)}{\partial \mu} & =\lambda_{\mu}^{r} \sum_{i}\left(S_{i}-\left(1+r+S_{i}-R_{i}\right) \cdot \Phi\left(\bar{x}^{i}\right)\right) \frac{\partial \alpha_{i}^{r}(\mu)}{\partial \mu} \\
& =-\lambda_{\mu}^{r}
\end{aligned}
$$

onde a última igualdade é obtida derivando a restrição de (P2') em relação a $\mu$.

Pelo Teorema do Envelope, a otimização (P1') nos fornece

$$
P M_{\alpha_{i}}^{r}=\left(S_{i}-\left(1+r+S_{i}-R_{i}\right) . \Phi\left(\frac{\bar{x}^{i}-\sqrt{\rho_{i}} Z^{i}}{\sqrt{1-\rho_{i}}}\right)\right)
$$

conseqüentemente obtemos

$$
\begin{aligned}
Z^{i} & =\frac{\bar{x}^{i}}{\sqrt{\rho_{i}}}-\sqrt{\frac{1-\rho_{i}}{\rho_{i}}} \cdot \Phi^{-1} \\
& \left(\frac{\left(S_{i}-\lambda_{\mu}^{r}\left(S_{i}-\left(1+r+S_{i}-R_{i}\right) . \Phi\left(\bar{x}^{i}\right)\right)\right)}{1+r+S_{i}-R_{i}}\right) \equiv h_{i}^{r}\left(\lambda_{\mu}^{r}\right)
\end{aligned}
$$

Substituindo na equação de restrição de (P1') obtemos $h_{i}^{r}\left(\lambda_{\mu}^{r}\right)^{\prime} \sum^{-1} h_{i}^{r}\left(\lambda_{\mu}^{r}\right)=$ $\chi_{1 \%}$. Portanto, $\lambda_{\mu}^{r}$ é uma constante que independe de $\mu$. Observando (AI.10), concluimos que o gráfico de $\operatorname{Risco}(\mu)$ é uma reta. Além disso, é óbvio que a solução de (P2) satisfaz à restrição da minimização (P2') e, conseqüentemente, $\operatorname{Risco}^{r}(\mu) \leq \operatorname{risco}(\mu)$.

As C.P.O. de (P1') são

$$
\begin{aligned}
& \alpha_{i} \cdot\left(1+r+S_{i}-R_{i}\right) \cdot \sqrt{\frac{\rho_{i}}{1-\rho_{i}}} \cdot \phi\left(\frac{\bar{x}^{i}-\sqrt{\rho_{i} h_{i}^{r}}}{\sqrt{1-\rho_{i}}}\right) \\
& \quad=-\lambda_{P 1} \cdot e_{i}^{\prime} \sum^{-1} h_{i}^{r}
\end{aligned}
$$


onde $\lambda_{P 1} \geq 0$ é o multiplicador de Kent-Tucker da restrição em (P1'), $\phi($. é a função de densidade da distribuição normal e $e_{i}$ é o vetor $n$ dimensional com 1 no $i$-ésimo componente e zero nos outros.

$\mathrm{O}$ vetor $h_{i}^{r}=h_{i}^{r}\left(\lambda_{\mu}^{r}\right)$ é constante e independe de $\mu$, assim como $\lambda_{\mu}^{r}$. Portanto $\alpha_{i}=c_{i} \cdot \lambda_{P 1}(\mu)$ onde $c_{i}=\frac{1}{\left(1+r+S_{i}-R_{i}\right) \cdot \sqrt{\frac{\rho_{i}}{1-\rho_{i}}} \cdot \phi\left(\frac{\bar{x}^{i}-\sqrt{\rho_{i} h_{i}^{r}}}{\sqrt{1-\rho_{i}}}\right)} e_{i}^{\prime} \sum^{-1} h_{i}^{r}$ independe de $\mu$.

Substituindo na restrição de (P2) obtemos $\quad \lambda_{P 1}(\mu)=\frac{\mu-r}{\sum_{i} c_{i}\left(v_{i}-r\right)}$ onde $v_{i}=r+S_{i}-\left(1+r+S_{i}-R_{i}\right) . \Phi\left(\bar{x}^{i}\right)$ é o retorno esperado do ativo $i$.

Logo, $\alpha_{i}=\frac{c_{i}}{\sum_{i} c_{i}\left(v_{i}-r\right)}(\mu-r)$ e $\sum_{i} \alpha_{i}=\frac{\sum_{i} c_{i}}{\sum_{i} c_{i}\left(v_{i}-r\right)}(\mu-r)$, funções afim de $\mu$. Existe então um único $\mu^{*}$ tal que a solução $\alpha^{*}$ de (P2') satisfaz $\sum_{i} \alpha_{i}^{*}=1$. É óbvio que $\alpha^{*}$ é também solução de $(\mathrm{P} 2)$ e $\operatorname{Risco}^{r}\left(\mu^{*}\right)=$ $\operatorname{Risco}\left(\mu^{*}\right)$. Além disso, se $\mu \neq \mu^{*}$ então $\sum_{i} \alpha_{i} \neq 1, \alpha$ não é factível para (P2) e $\operatorname{Risco}^{r}(\mu)<\operatorname{Risco}(\mu)$. Portanto, $\operatorname{Risco}^{r}(\mu)$ é a reta tangente a $\operatorname{Risco}(\mu)$ em $\mu^{*}$.

Por fim, como $\alpha_{i}=\frac{c_{i}}{\sum_{i} c_{i}\left(v_{i}-r\right)}(\mu-r)$ é uma função afim de $\mu$., qualquer portfólio ótimo pode ser escrito como a combinação linear de $\alpha^{*}$ e do ativo sem risco.

Teorema 3:

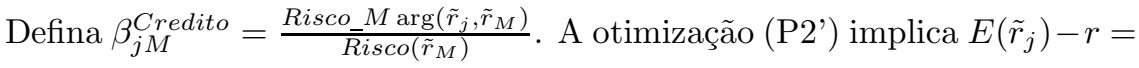
$\beta_{j M}^{\text {Credito }}\left[E\left(\tilde{r}_{M}\right)-r\right]$, versão do modelo CAPM para ativos de crédito.

Demonstração: Seja $\alpha^{M}$ o portfólio de mercado. Este portfólio está na fronteira ótima de (P2') pois é uma combinação linear de portfólios ótimos das várias instituições financeiras. Sabe-se que

$$
\tilde{r}_{j}=r+S_{j}-\left(1+r+S_{j}-R_{j}\right) \Phi\left(\frac{\bar{x}^{j}-\sqrt{\rho_{j}} Z^{j}}{\sqrt{1-\rho_{j}}}\right)
$$

e $\tilde{r}_{M}=\sum_{j} \alpha_{j}^{M} \tilde{r}_{j}-\left(1-\sum_{i} \alpha_{j}^{M}\right) . r$

$\log$,

$$
\begin{array}{r}
E\left(\tilde{r}_{j}\right)-r=S_{j}-\left(1+r+S_{j}-R_{j}\right) \Phi\left(\bar{x}^{j}\right) \\
\text { e } E\left(\tilde{r}_{M}\right)-r=\sum_{j} \alpha_{j}^{M}\left[S_{j}-\left(1+r+S_{j}-R_{j}\right) \Phi\left(\bar{x}^{j}\right)\right] .
\end{array}
$$


Por outro lado, as condições de primeira ordem do programa (P2') e o Teorema do Envelope aplicado a (P1') fornecem

$$
P M_{\alpha_{i}}^{r}=\lambda_{\mu}^{r}\left(S_{i}-\left(1+r+S_{i}-R_{i}\right) \cdot \Phi\left(\bar{x}^{i}\right)\right)
$$

e $P M_{\alpha_{i}}^{r}=\left(S_{i}-\left(1+r+S_{i}-R_{i}\right) \cdot \Phi\left(\frac{\bar{x}^{i}-\sqrt{\rho_{i}} Z_{j}^{*}}{\sqrt{1-\rho_{i}}}\right)\right)$

onde $\lambda_{\mu}^{r}$ é o multiplicador de Lagrange da restrição em (P2') e $Z^{*}=$ $\left[Z_{1}^{*}, Z_{2}^{*}, \ldots\right]$ o ótimo em (P1') . Portanto,

$$
\begin{aligned}
\operatorname{Risco}{ }_{-} M \arg \left(\tilde{r}_{j}, \tilde{r}_{M}\right)= & S_{j}-\left(1+r+S_{j}-R_{j}\right) \Phi \\
& \left(\frac{\bar{x}^{j}-\sqrt{\rho_{j}} Z_{j}^{*}}{\sqrt{1-\rho_{j}}}\right) \\
= & \lambda_{\mu}^{r}\left(S_{i}-\left(1+r+S_{i}-R_{i}\right) \cdot \Phi\left(\bar{x}^{i}\right)\right)
\end{aligned}
$$

e

$$
\begin{aligned}
\operatorname{Risco}\left(\tilde{r}_{M}\right) & =\sum \alpha_{j}^{M}\left[S_{j}-\left(1+r+S_{j}-R_{j}\right) \Phi\left(\frac{\bar{x}^{j}-\sqrt{\rho_{j}} Z_{j}^{*}}{\sqrt{1-\rho_{j}}}\right)\right] \\
& =\lambda_{\mu}^{r} \sum_{j} \alpha_{j}^{M}\left[S_{j}-\left(1+r+S_{j}-R_{j}\right) \Phi\left(\bar{x}^{j}\right)\right]
\end{aligned}
$$

Assim, por simples álgebra,

$$
\begin{aligned}
\beta_{j M}^{\text {Credito }} & =\frac{\text { Risco_M } \arg \left(\tilde{r}_{j}, \tilde{r}_{M}\right)}{\operatorname{Risco}\left(\tilde{r}_{m}\right)} \\
& =\frac{\lambda_{\mu}^{r}\left[S_{j}-\left(1+r+S_{j}-R_{j}\right) \Phi\left(\bar{x}^{j}\right)\right]}{\lambda_{\mu}^{r} \sum_{j} \alpha_{j}^{M}\left[S_{j}-\left(1+r+S_{j}-R_{j}\right) \Phi\left(\bar{x}^{j}\right)\right]} \\
& =\frac{E\left(\tilde{r}_{j}\right)-r}{E\left(\tilde{r}_{M}\right)-r}
\end{aligned}
$$

como queria-se demonstrar.

\section{Apêndice II. Cálculo dos Momentos da Variável Aleatória $Y$.}

Suponha que $Y$ é dada pela equação (3) onde $Z$ é uma v.a. com distribuição normal padrão. Já demonstramos na proposição 1 que $Y$ é o limite em distribuição da seqüência $\left\{Y_{n}\right\}, n=1,2,3, \ldots$ onde cada elemento é 
dado pela equação (2). Observe que sob a hipótese de independência entre as v.as. $\left\{Z, \varepsilon_{1}, \varepsilon_{2}, \varepsilon_{3}, \varepsilon_{4}, \ldots\right\}$, as v.as. $\left\{X_{i}\right\}_{i=1,2,3, \ldots}$ das equações (1) têm distribuição normal padrão. Além disso, qualquer par destas v.as. apresentam correlação igual a $\rho$. Com isso, é fácil verificar que

$$
\begin{aligned}
E\left[\chi_{i 1} \chi_{i 2} \ldots \chi_{i n}\right] & =P\left(X_{i 1} \leq \bar{x}, X_{i 2} \leq \bar{x}, \ldots, X_{i n} \leq \bar{x}\right) \\
& =\Phi_{n}(\bar{x}, \bar{x}, \ldots, \bar{x}, \rho)
\end{aligned}
$$

onde $\Phi_{n}(., ., \ldots, ., \rho)$ representa a função de distribuição Gaussiana conjunta acumulada de dimensão $n$ com média $[0,0, \ldots, 0]^{t}$ e matriz de Variância Covariância

$$
\left[\begin{array}{cccc}
1 & \rho & \cdots & \rho \\
\rho & 1 & \rho & \vdots \\
\vdots & \rho & \ddots & \rho \\
\rho & \cdots & \rho & 1
\end{array}\right] .
$$

Observe que é possível calcular a probabilidade no centro da equação (AII.1) de uma maneira alternativa. Condicionando em $Z,\left\{X_{i} \mid Z\right\}_{i=1,2,3}$ são v.as independentes e identicamente distribuídas. Conseqüentemente

$$
\begin{gathered}
P\left(X_{i_{1}} \leq \bar{x}, X_{i_{2}} \leq \bar{x}, \ldots, X_{i_{n}} \leq \bar{x}\right)= \\
\int_{-\infty}^{\infty} P\left(X_{1} \leq \bar{x} \mid Z\right)^{n} F_{z}(d z)
\end{gathered}
$$

Porém, $\left\{X_{i} \mid Z\right\}_{i=1,2,3, \ldots}$ têm distribuição normal com média $\sqrt{\rho Z}$ e variância $1-\rho$, então

$$
P\left(X_{1} \leq \bar{x} \mid Z\right)=\Phi\left(\frac{\bar{x}-\sqrt{\rho Z}}{\sqrt{1-\rho}}\right)
$$

Finalmente, substituindo a equação (AII.3) na equação (AII.2), podemos notar que o lado esquerdo da equação (AII.2) representa o momento de ordem $n$ da v.a. $Y$. Utilizando o resultado da equação (AII.1) obtemos

$$
E\left[Y^{n}\right]=\int_{-\infty}^{\infty} P\left(X_{1} \leq \bar{x} \mid Z\right)^{n} F_{z}(d z)
$$

\section{Apêndice III. Cálculo do VAR Usando a Taxa de Inadimplência Limite $Y$.}


O Valor a risco (V.A.R.) de $W$ com nível de confiança de $\alpha \%$ satisfaz à equação $P(W \leq \operatorname{VaR}(\alpha))=\alpha$

Pela equação (4) e observando que $R-S-r-1<0$, obtemos

$$
P\left(Y \geq \frac{\operatorname{VaR}(\alpha)-S-r}{R-S-r-1}\right)=\alpha
$$

Utilizando a definição de $Y$ da equação (3), obtemos

$$
P\left(Z \leq \frac{\bar{x}-\sqrt{(1-\rho)} \Phi^{-1}\left(\frac{V a R(\alpha)-S-r}{R-S-r-1}\right)}{\sqrt{\rho}}\right)=\alpha
$$

Denotando por $F_{z}($.$) a distribuição de probabilidade acumulada da v.a. Z$, podemos inverter a equação anterior para obter

$$
\frac{\bar{x}-\sqrt{(1-\rho)} \Phi^{-1}\left(\frac{V a R(\alpha)-S-r}{R-S-r-1}\right)}{\sqrt{\rho}}=F_{z}^{-1}(\alpha)
$$

Finalmente, um pouco de álgebra nos dá

$$
\operatorname{VaR}(\alpha)=(R-S-r-1) \Phi\left(\frac{\bar{x}-F_{z}^{-1}(\alpha) \sqrt{\rho}}{\sqrt{1-\rho}}\right)+S+r
$$

Considere agora o caso onde aproximamos $Y$ por uma distribuição normal com primeiro e segundo momento iguais ao primeiro e segundo momento da v.a. da equação (3). Isto é, com média e variância $E[Y]=\Phi(\bar{x})$ e $\sigma_{Y}^{2}=E\left[(Y-E(Y))^{2}\right]=\Phi_{2}(\bar{x}, \bar{x}, \rho)-(\Phi(\bar{x}))^{2}$, onde $\Phi_{2}(., ., \rho)$ é a função de distribuição acumulada da normal bivariada. Assim,

$$
P\left(\frac{Y-\phi(\bar{x})}{\sigma_{Y}}<\frac{\frac{V a R(\alpha)-S-r}{R-S-r-1}-\Phi(\bar{x})}{\sigma_{Y}}\right)=1-\alpha
$$

conseqüentemente

$$
\operatorname{NormalVaR}(\alpha)=(R-S-r-1)\left(\sigma_{Y} \Phi^{-1}(1-\alpha)+\Phi(\bar{x})\right)+S+r
$$

